\title{
Recursos eletrotermofototerapêuticos no tratamento da fibromialgia*
}

\author{
Electrothermal and phototherapeutic resources to treat fibromyalgia \\ Lucas Lima Ferreira ${ }^{1}$, Laís H. Carvalho Marino², Simone Cavenaghi \\ * Recebido do Departamento de Ciências Neurológicas da Faculdade de Medicina de São José do Rio Preto \\ (FAMERP). São José do Rio Preto, SP.
}

\section{RESUMO}

JUSTIFICATIVA E OBJETIVOS: A fibromialgia (FM) é uma síndrome de etiologia desconhecida, de caráter crônico e não articular, com característica típica de dor difusa pelo corpo, presença de tender points específicos, fadiga muscular, distúrbios do sono, sintomas depressivos e queixas cognitivas. A eletrotermofototerapia é muito utilizada na prática clínica da fisioterapia como parte do programa global de reabilitação para a redução dos sintomas da FM. Este estudo objetivou agrupar e atualizar conhecimentos em relação aos recursos eletrotermofototerapêuticos que têm sido empregados para o tratamento de pacientes portadores de FM.

CONTEÚDO: Foi realizada pesquisa da literatura nas bases de dados eletrônicas MedLine, LILACS, Cochrane, PubMed e Scielo, no período de janeiro de 2005 a dezembro de 2010, sendo encontrados sete artigos potencialmente relevantes. Entre os estudos, dois utilizaram o laser de baixa potência, quatro utilizaram a esti-

1. Fisioterapeuta Especialista em Aprimoramento em Fisioterapia do Hospital de Base e Faculdade de Medicina de São José do Rio Preto (FUNFARME/FAMERP). São José do Rio Preto, SP, Brasil.

2. Fisioterapeuta do Hospital de Base e Faculdade de Medicina de São José do Rio Preto (FUNFARME/FAMERP); Doutoranda em Ciências da Saúde pela Faculdade de Medicina de São José do Rio Preto (FAMERP); Chefe do Serviço de Fisioterapia FUNFARME/FAMERP. São José do Rio Preto, SP, Brasil.

3. Fisioterapeuta do Hospital de Base e Faculdade de Medicina de São José do Rio Preto (FUNFARME/FAMERP); Doutora em Ciências da Saúde pela Faculdade de Medicina de São José do Rio Preto (FAMERP). São José do Rio Preto, SP, Brasil.

Endereço para correspondência:

Lucas Lima Ferreira

R. Prof ${ }^{\circ}$ Enjolrrás Vampré, 190/33 - Vila Santa Cândida

15091-290 São José do Rio Preto, SP.

Fone: (17) 9183-7915

E-mail: lucas_lim21@hotmail.com mulação elétrica e um usou o ultrassom terapêutico. Os recursos eletrotermofototerapêuticos têm sido cada vez mais empregados no tratamento do principal sintoma da FM, a dor.

CONCLUSÃO: Os recursos eletrotermofototerapêuticos têm sido ferramenta constante no tratamento da FM, no entanto novos recursos como as correntes de estimulação transcraniana têm surgido, mas as generalizações quanto aos benefícios da eletrotermofototerapia, efeitos adversos e doses no tratamento da FM ainda necessitam de novos estudos com maior rigor metodológico.

Descritores: Dor, Fibromialgia, Modalidades de fisioterapia.

\section{SUMMARY}

BACKGROUND AND OBJECTIVES: Fibromyalgia (FM) is a syndrome of unknown etiology, chronic and non-articular, with typical features of pain diffused through the body, presence of specific tender points, muscle fatigue, sleep disorders, depressive symptoms and cognitive complaints. Electrothermal and phototherapy are widely used in physical therapy as part of the global rehabilitation program to decrease FM symptoms. This study aimed at grouping and updating knowledge on electrothermal and phototherapeutic resources used to treat FM patients.

CONTENTS: Literature was researched in the electronic databases MedLine, LILACS, Cochrane, PubMed and Scielo, from January 2005 to December 2010, being found seven potentially relevant studies. Among them, two have used low potency laser, four have used electrical stimulation and one has used therapeutic ultrasound. Electrothermal and phototherapeutic resources have been increasingly used to treat pain, which is the primary FM symptom.

CONCLUSION: Electrothermal and phototherapeutic resources have been a constant tool to treat FM, howe- 
ver new resources, such as transcranial stimulation currents have appeared. Generalizations about the benefits of electrothermal and phototherapy, adverse effects and doses to treat FM still need further studies with higher methodological rigor.

Keywords: Fibromyalgia, Pain, Physical therapy modalities.

\section{INTRODUÇÃO}

A fibromialgia (FM) é uma síndrome de etiologia desconhecida, de caráter crônico e não articular, com característica típica de dor difusa pelo corpo, presença de tender points específicos, fadiga muscular, distúrbios do sono, sintomas depressivos e queixas cognitivas ${ }^{1,2}$. Segundo os critérios do Colégio Americano de Reumatologia ${ }^{1}$, desde 1990 foi adotado como padrão pela comunidade científica internacional especializada, o diagnóstico de fibromialgia, que exige pelo menos três meses consecutivos de dor generalizada e dor à palpação, com cerca de $4 \mathrm{~kg}$ de pressão pela ponta do dedo, em no mínimo 11 de 18 pontos pré-definidos.

Pouco se conhece sobre a etiologia e patogênese da $\mathrm{FM}^{3,4}$. Estudos recentes sugerem uma origem multifatorial na qual ansiedade prolongada e estresse emocional, traumas, doenças ocupacionais, interrupção repentina de medicamentos, hipertireoidismo e infecções podem ser fatores de risco para o início da doença ${ }^{3,4}$. Em relação a epidemiologia, a FM é mais prevalente no sexo feminino e entre a faixa etária dos 35-60 anos 5 .

Pelo caráter crônico e de múltiplos sintomas da FM, o tratamento recomendado aos seus portadores é baseado na abordagem interdisciplinar, com intervenções no âmbito físico, farmacológico, cognitivo-comportamental e educacional $^{6}$. No âmbito das intervenções físicas, a fisioterapia se destaca pela riqueza de modalidades terapêuticas como cinesioterapia, hidroterapia, eletrotermofototerapia, relaxamento, massoterapia, acupuntura e outros, que podem ser utilizadas no controle da $\mathrm{FM}^{7}$.

A eletrotermofototerapia (ETFT) é muito utilizada na prática clínica da fisioterapia, e sua efetividade foi observada em revisão sobre desordens osteomioarticulares $^{8}$. As intervenções por meio da ETFT são utilizadas como parte do programa global de reabilitação, principalmente para alívio da dor ${ }^{6}$, o que leva consequentemente, a aumento na amplitude de movimento, força muscular, mobilidade, resistência física, habilidade de andar e estado funcional ${ }^{7}$. Além disso, esses recursos oferecem muitas vantagens, pois são intervenções não invasivas e rápidas de administrar, resultando em poucos efeitos adversos e contraindicações, quando comparadas com as intervenções farmacológicas para a redução dos sintomas da $\mathrm{FM}^{7}$.

Com base no exposto, este estudo objetivou agrupar e atualizar conhecimentos em relação aos recursos eletrotermofototerapêuticos que têm sido empregados para o tratamento de pacientes portadores de fibromialgia.

\section{CONTEÚDO}

A pesquisa da literatura foi realizada nas bases de dados eletrônicas: MedLine, LILACS, Cochrane, PubMed e Scielo, no período de janeiro de 2005 a dezembro de 2010, cruzando o descritor fibromyalgia com electrotherapy modalities, electrotherapy, ultrasound, phototherapy, laser therapy, interferencial current, transcutaneous electrical stimulation, thermotherapy, physiotherapy.

A busca foi limitada nas linguagens inglesa, espanhola ou portuguesa, com estudos realizados em humanos adultos de 18 anos ou mais. Não foram incluídos na pesquisa resumos de dissertações ou teses acadêmicas.

Foi realizada uma análise de títulos e resumos para obtenção de estudos, sendo encontrados sete artigos potencialmente relevantes.

\section{Recursos eletrotermofototerapêuticos e fibromialgia} Existe um grande número de modalidades eletrofototerapêuticas utilizadas na prática clínica da fisioterapia com pacientes fibromiálgicos, sendo consideradas como recursos que, em conjunto, devem compor o tratamento desses pacientes para se obterem resultados satisfató$\operatorname{rios}^{7,8}$.

O laser de baixa potência é amplamente utilizado em pacientes com desordens osteomioarticulares e os efeitos terapêuticos desse tipo de laser são a ação anti-inflamatória, a analgesia e a modulação da atividade celular ${ }^{9}$. Para a FM o laser é recomendado, principalmente para o alívio da dor. Como a dor crônica está intimamente relacionada com os outros sintomas da FM, acredita-se que sua redução causaria um efeito cascata para a melhora dos demais sintomas? ${ }^{7}$.

Em ensaio clínico aleatório controlado com 20 pacientes do sexo feminino, média de idade de 45 anos, portadoras de $\mathrm{FM}^{10}$, os desfechos avaliados foram a dor, pelo limiar através da pressão exercida pelo dolorímetro nos tender points e pela intensidade geral mensurada pela escala analógica visual (EAV); e a qualidade de vida (QV), avaliada pelo questionário Short Form 36 (SF-36). As pacientes foram divididas em dois grupos: o experimen- 
tal foi realizado aplicação de laser GAAlAs, 830 nanômetros $(\mathrm{nm})$, potência $30 \mathrm{mw}$, com aplicação contínua de $3 \mathrm{~J} / \mathrm{cm}^{2}$ em cada tender point associada a exercícios de alongamentos gerais; o grupo controle recebeu apenas exercícios de alongamentos gerais. Ambos os grupos foram tratados durante cinco semanas num total de 10 sessões. Verificou-se melhora significativa da dor pela EAV e da QV pelo SF-36 em ambos os grupos e não houve diferença entre os grupos quanto às medidas após as intervenções.

Outro ensaio clínico aleatório e controlado aplicou o laser em 32 pacientes fibromiálgicas do sexo feminino com idade média de 38 anos. Os autores avaliaram a dor, por meio da Escala de Likert e um escore específico, e a QV, através do Fibromyalgia Impact Questionnaire (FIQ). Dois grupos receberam aplicação do laser GAAlAs, $830 \mathrm{~nm}$, potência $50 \mathrm{mw}$, com emissão de $2 \mathrm{~J}$ por um minuto em cada tender point, cinco vezes por semana durante 10 sessões, sendo que o grupo controle (GC) placebo recebeu o mesmo procedimento sem a emissão dos raios. Os resultados apontaram melhora significativa de todos os parâmetros avaliados no grupo experimental (GE) após a intervenção e no seguimento em relação ao início, no FIQ, bem estar subjetivo e escore para a dor no GE em relação ao GC após a intervenção ${ }^{11}$.

A estimulação elétrica pode trazer inúmeros benefícios quando indicada no controle da dor, pois, com a sua redução, o paciente aumenta o seu nível de função e atividade, pode participar de programas de exercícios físicos e melhorar a sua $\mathrm{QV}^{12}$. A estimulação elétrica nervosa transcutânea (TENS) tem como ação mais reconhecida a analgesia, que ocorre por meio de corrente elétrica de baixo limiar que inibe a transmissão dos estímulos dolorosos na medula espinhal e liberam opioides endógenos, como as endorfinas ${ }^{13,14}$.

Silva e col. ${ }^{15}$ realizaram ensaio clínico aleatório para avaliar os níveis de dor pela EAV, de QV pelo SF-36 e de depressão pelo Inventário de Beck, com 10 mulheres portadoras de FM com idade média entre 47 e 50 anos. Foram formados dois grupos, no GE foi aplicado TENS com a utilização de eletrodos de superfície e gel condutor posicionado nos tender points dos músculos do trapézio, supraespinhoso, glúteo e interlinha medial do joelho bilateralmente, com os parâmetros frequência de pulso de $15 \mathrm{~Hz}$, tempo de pulso de $150 \mu$ s e intensidade pela sensação de formigamento, durante 40 minutos, três vezes por semana; o GC foi submetido a hidroterapia composta por aquecimento (5 min.), alongamentos (20 min.) e exercícios aeróbicos (15 min.). Houve melhora da dor, dos sintomas depressivos e da qualidade de vida no GE, melhora da QV no GC e após a intervenção o GE apresentou menor EAV em relação ao GC.

Estudo realizado com 66 pacientes portadores de FM utilizou a eletroacupuntura (EA) $(\mathrm{n}=22)$, a estimulação elétrica dermoneurológica (EEDN) (aplicada a pontos de acupuntura) $(\mathrm{n}=22)$ ou oryzanol, vitamina B1 e amitriptilina $(\mathrm{n}=22)$ diariamente por 45 dias. A EAV foi utilizada para avaliar a dor, outros sintomas e adaptabilidade social antes e após cada tratamento e em 45 dias pós-tratamento. Houve pouca diferença entre os grupos EEDN e EA, mas quando comparado com o terceiro grupo, houve uma diferença significativa $(\mathrm{p}<$ 0,01). Não houve desistências. Aos 24 meses, as taxas de recorrência dos grupos EA e EEDN foram inferiores às do terceiro grupo. Convém, no entanto, notar que a taxa de recorrência não é uma medida de resultado aceita para fibromialgia ${ }^{16}$.

Estudo realizado com quatro mulheres com FM, depressão e transtorno de personalidade utilizando estimulação magnética transcraniana repetitiva (EMTr) com o objetivo de observar um novo tratamento antidepressivo, que também pode ser útil no tratamento da dor crônica ${ }^{17}$. As pacientes receberam $1 \mathrm{~Hz}$ de EMTr aplicado ao córtex dorsolateral pré-frontal direito. As participantes classificavam a dor usando a escala de 11 pontos de Likert. A média de dor pré-tratamento foi 8,2 e reduziu para 1,5 pontos após o tratamento $(\mathrm{p}<0,009)$. Todas tiveram melhora da dor e duas tiveram resolução completa da dor. Apenas uma teve resposta antidepressiva. Estes resultados preliminares sugerem um possível papel para a EMTr no tratamento da FM.

A estimulação transcraniana por corrente contínua (ETCC) pode modular a atividade do cérebro de forma não invasiva e pode diminuir a dor em pacientes com dor central refratária ${ }^{18}$. Com base nessa afirmação, Fregni e col. ${ }^{18}$ avaliaram a hipótese de que o tratamento com ETCC resultaria em alívio da dor em pacientes com FM. Trinta e dois pacientes foram aleatorizados para receber estimulação simulada ou ETCC real, $2 \mathrm{~mA}$ por $20 \mathrm{minu}$ tos em 5 dias consecutivos, com o ânodo centrado sobre o córtex motor primário (M1) ou no córtex pré-frontal dorsolateral (CPFDL). Um avaliador cego avaliou a dor do paciente utilizando a EAV, a impressão global do clínico, a avaliação global do paciente e o número de pontos dolorosos. Outros sintomas da FM foram avaliados usando o FIQ e o SF-36. A segurança foi avaliada com uma bateria de testes neuropsicológicos. A ETCC anódica do córtex motor primário induziu melhora significativamente maior da dor em comparação com estimulação 
simulada e a estimulação do CPFDL $(p<0,0001)$. Embora esse efeito diminuiu após o término do tratamento, ainda foi significativo após três semanas de seguimento $(\mathrm{p}=0,004)$. Um pequeno impacto positivo na $\mathrm{QV}$ foi observada entre os pacientes que receberam a estimulação anódica M1. Esses resultados fornecem evidência inicial de um efeito benéfico da ETCC na fibromialgia, estimulando novos estudos.

O ultrassom (US) terapêutico é utilizado pela fisioterapia por seus efeitos fisiológicos decorrentes tanto de sua ação mecânica como térmica. A ação mecânica aumenta a permeabilidade celular, diminui a resposta inflamatória, reduz a dor por meio da diminuição da velocidade de condução nas fibras nervosas e facilita o processo de cicatrização dos tecidos moles. O US contínuo tem ação térmica que contribui para o aumento da vasodilatação local e melhora da inflamação crônica, reduz o espasmo muscular e a dor ${ }^{19}$.

Estudo de coorte observacional prospectivo com objetivo de avaliar a curto prazo e após um ano, os resultados da manipulação do tecido conjuntivo combinado com US terapêutico em termos de dor, queixa de sono não reparador, e o impacto sobre as atividades funcionais através da EAV, realizado em 20 pacientes do sexo feminino com $\mathrm{FM}^{20}$. Todas as avaliações foram realizadas antes e após 20 sessões de tratamento, que incluiu a manipulação do tecido conjuntivo do dorso diária combinada ao US terapêutico na região superior das costas. Após um ano de acompanhamento foram realizadas avaliações em 14 indivíduos. O teste de Friedman foi utilizado para analisar as mudanças dependentes do tempo. A análise estatística revelou que a intensidade da dor, o impacto da FM em atividades funcionais, e queixas de sono não reparador melhoraram após o programa de tratamento $(\mathrm{p}<0,005)$. Os métodos utilizados neste estudo parece ser úteis para melhorar a intensidade da dor, queixas de sono não reparador e o impacto sobre as atividades funcionais em pacientes com FM.

Os recursos eletrotermofototerapêuticos têm sido ferramenta constante, por sua reconhecida ação analgésica, no tratamento de pacientes portadores de fibromialgia. Entre esses recursos, os mais tradicionais como laser, ultrassom e TENS, continuam tendo aplicação comum no dia a dia do terapeuta. Entretanto, novos recursos como as correntes de estimulação magnética transcraniana repetitiva e de estimulação transcraniana por corrente contínua têm surgido na busca de um tratamento mais global e que enfoque todos os sintomas inerentes desta doença. Porém, generalizações quanto aos benefícios da eletro- termofototerapia, efeitos adversos e doses do tratamento na FM ainda são restritas.

\section{CONCLUSÃO}

Os recursos eletrotermofototerapêuticos têm sido ferramenta constante no tratamento da FM, no entanto novos recursos como as correntes de estimulação transcraniana têm surgido, mas as generalizações quanto aos benefícios da eletrotermofototerapia, efeitos adversos e doses no tratamento da FM ainda necessitam de novos estudos com maior rigor metodológico.

\section{REFERÊNCIAS}

1. Wolfe F, Smythe HA, Yunus MB, et al. The american college of reumatology criteria for the classification of fibromyalgia. Report of the multicenter criteria committee. Arthritis Rheum 1990;33(2):160-72.

2. Agnol LD, Martelete M. Hidroterapia no tratamento de pacientes com fibromialgia. Rev Dor 2009;10(3):250-4.

3. Consalter E, Sanches ML, Guimarães AS. Correlação entre disfunção temporomandibular e fibromialgia. Rev Dor 2010;11(3):237-41.

4. Heymann R, Paiva E, Helfeinstein M. et al. Consenso brasileiro do tratamento da fibromialgia. Rev Bras Reumatol 2010;50(1):56-60.

5. Cavalcante AB, Sauer JF, Chalot SD, et al. A prevalência de fibromialgia: uma revisão de literatura. Rev Bras Reumatol 2006;46(1):40-8.

6. Gür A. Physical therapy modalities in management of fibromyalgia. Curr Pharm Des 2006;12(1):29-35.

7. Ricci NA, Dias CNK, Driusso P. The use of electrothermal and phototherapeutic methods for the treatment of fibromyalgia syndrome: a systematic review. Rev Bras Fisioter 2010;14(1):1-9.

8. Ottawa P. Ottawa Panel evidence based clinical practice guidelines for electrotherapy and thermotherapy interventions in the management of rheumatoid arthritis in adults. Phys Ther 2004;84(11):1016-43.

9. Fukuda TY, Malfatti CA. Análise da dose do laser de baixa potência em equipamentos nacionais. Rev Bras Fisioter 2008;12(1):70-4.

10. Matsutani LA, Marques AP, Ferreira EA, et al. Effectiveness of muscle stretching exercises with and without laser therapy at tender points for patients with fibromyalgia. Clin Exp Rheumatol 2007;25(3):410-5.

11. Armagan O,Tascioglu F, Ekim A, et al. Long-term efficacy of low level laser therapy in women with fi- 
bromyalgia: a placebo-controlled study. J Back Musculoskelet Rehabil 2006;19:135-40.

12. Ferreira LL, Cavenaghi S, Marino LHC. Recursos eletroterapêuticos no tratamento da dor oncológica. Rev Dor 2010;11(4):339-42.

13. Resende MA, Golçalves HH, Sabino GS, et al. Redução do efeito analgésico da estimulação elétrica nervosa transcutânea de baixa freqüência em ratos tolerantes à morfina. Rev Bras Fisioter 2006;10(3):291-6.

14. Abreu EA, Santos JDM, Ventura PL. Efetividade da eletroestimulação nervosa transcutânea no alívio da dor durante o trabalho de parto: um ensaio clínico controlado. Rev Dor 2010;11(4):313-8.

15. Silva TFG, Suda EY, Marçulo CA, et al. Comparação dos efeitos da estimulação elétrica nervosa transcutânea e da hidroterapia na dor, flexibilidade e qualidade de vida de pacientes com fibromialgia. Fisioter Pesq 2008;15(2):118-24.

16. Guo X, Jia J. Comparison of therapeutic effects on fibromyalgia syndrome between dermal-neurological electric stimulation and electric acupuncture. Chinese J Clin Rehab 2005;9(1):171-3.

17. Sampson M, Rome JD, Rumamans TA. Slow-frequency rTMS reduces fibromyalgia pain. Pain Med 2006;7(2):115-8.

18. Fregni F, Gimenes R, Valle AC, et al. A randomized, sham-controlled proof of principle study of transcranial direct current stimulation for the treatment of pain in fibromyalgia. Arthritis Rheum 2006;54(12):3988-98.

19. Maggi LE, Omena TP, Von Krüger MA, et al. Software didático para modelagem do padrão de aquecimento dos tecidos irradiados por ultrassom fisioterapêutico. Rev Bras Fisioter 2008;12(3):204-14.

20. Citak-Karakaya I, Akbayrak T, Demirtürk F, et al. Short and long-term results of connective tissue manipulation and combined ultrasound therapy in patients with fibromyalgia. J Manipulative Physiol Ther 2006;29(7):524-8.

Apresentado em 28 de junho de 2011.

Aceito para publicação em 01 de setembro de 2011.

Conflito de interesses: Nenhum. 